3. Dignass, et al. J Crohn's Colitis 2015;9(3):211-22.

\section{PTU-125 IMPROVING QUALITY AND REDUCING FREQUENCY OF HOSPITAL CARE FOR ALCOHOL-USE DISORDER PATIENTS: A MULTI-DISCIPLINARY-TEAM APPROACH}

${ }^{1}$ Lynn Owens*, ${ }^{1}$ Cecil Kullu, 'David Byrne, ${ }^{1}$ Elaine Lewis, ${ }^{1}$ Kev Patterson, ${ }^{1}$ Paul Richardson. ${ }^{1}$ Royal Liverpool University Hospital Trust, Liverpool, UK ${ }^{2}$ University of Liverpool, Liverpool, UK

\subsection{6/gutjnl-2018-BSGAbstracts.503}

Introduction Patients with alcohol dependence often have complex health and social care needs resulting in frequent attendance at hospital. All too often care is aimed at optimising medical treatment of presenting conditions with little attention to, and planning for mitigation of causes, frequently exacerbated by non-medical problems. This failure to address the wider determinants of health often leads to a cycle of readmission. Therefore we aimed to improve the overall management of this patient group by bringing together a multidisciplinary team (MDT) to develop personalised multi-service, multi-professional care pathways.

Methods We developed a core multidisciplinary group with representation from our hospital hepatology team, our alcohol service, liaison psychiatry, occupational therapy, and our partners in primary care and homeless services. Other professionals and services were invited to the MDT meeting based on individual patient needs; this included the patient and or family and carers as appropriate. The purpose of this group was to develop a bespoke pathway of care with all current and future care providers, and foster an atmosphere for collaboration and mutual support. Our patients were often being cared for by multiple services, however much of this work was happening in isolation and was at times conflicting. Importantly, the patients were unclear where to go for what, and were utilising the ED as a failsafe when they were troubled.

Results MDT facilitated communication between services, professionals and the patient. This helped us provide planned rather than reactive care. For our 46 patients who have been presented at MDT, at six month follow-up we were able to demonstrate a significant reduction in hospital attendance and admission, resulting in $\sim 120$ less admissions and $\sim 434 \mathrm{ED}$ attendances across the acute trust; this equates to a saving within the last 6 months of an estimated $£ 63600$ on ED attendance alone.

Conclusions MDT meetings are a familiar element of system delivery within acute hospitals. What is unique about our approach, and has resulted in significant quality improvement is that we invested time building relationships with people from organisations not traditionally included in acute hospital care planning. This included those working in homeless shelters, probation services, voluntary agencies, families and patients. We believe our success could provide the confidence for other acute care teams across the NHS to replicate our model.

\section{PTU-126 IS THERE ANY ROLE FOR FLEXIBLE SIGMOIDOSCOPY FOR INPATIENTS WITH OVERT LOWER GASTROINTESTINAL BLEEDING?}

Mehul Patel*, Ezgi Ozcan, Aria Khani, Kalpesh Besherdas. Royal Free NHS Foundation Trust, London, UK

\subsection{6/gutjnl-2018-BSGAbstracts.504}

Introduction Inpatient flexible sigmoidoscopy (FS) is frequently requested to investigate overt lower gastrointestinal bleeding (LGIB), a condition where evidence based guidelines lack clarity. We sought to evaluate the benefit of FS in this setting, specifically: diagnostic yield, requirement for endoscopic therapy and comparison to diagnostic CT. Ultimately, to determine if FS is being over utilised.

Methods We retrospectively reviewed electronic healthcare records for all inpatients that underwent FS for LGIB (January 2016 - January 2018) at Barnet General Hospital. Recording the diagnosis on discharge; endoscopic findings; radiological findings and intervals between admission, endoscopy and discharge.

Results 87 inpatients underwent FS for LGIB (44 male and 43 female patients, mean age of 69 years (range=70)). Median length of stay was 6 days (range $=126$ ). The median duration from admission to FS and FS to discharge was 2 days $($ range $=125)$ and 3 days (range $=49$ ), respectively. Accounting for multiple pathologies in a single patient, documented discharge diagnoses included: diverticular disease (35.8\%), haemorrhoids (15.8\%) inflammatory bowel disease (7.4\%) malignancy $(5.3 \%)$ and infective colitis $(5.3 \%)$, no cause was found in $10.5 \%$. 46 (52.9\%) patients underwent a CT scan. Findings included: diverticular disease (31.3\%), colitis (19.7\%) and malignancy (4.9\%). FS findings included diverticular disease $(44.4 \%)$, colitis $(17.8 \%)$, haemorrhoids (15.6\%), polyps $(2.2 \%)$ and malignancy (1.1\%). $41.3 \%$ of CT scans were unremarkable. FS did not identify a cause in $66.7 \%$ of cases. $54.3 \%$ of findings on CT matched endoscopic findings. 2 $(2.0 \%)$ patients required surgery. $2(2.0 \%)$ patients required interventional radiology. 5 patients $(5.7 \%)$ required endoscopic therapy ( 2 APC for radiation proctitis, 1 haemorrhoid banding, 1 post-polypectomy bleed, 1 rectal packing), 4 (80.0\%) had active bleeding during FS; with no association with comorbidities or anticoagulation. $24(27.6 \%)$ of patients required blood transfusion. $23(26.0 \%)$ patients underwent outpatient colonoscopy.

Conclusions FS has limited diagnostic and therapeutic yield, identifying a cause for LGIB in a third of inpatients. Most patients did not require endoscopic therapy and were not actively bleeding during FS. Endoscopic therapy was more likely if bleeding was from a rectal source (radiation proctitis, haemorrhoids, recent polypectomy). We recommend inpatient pathways incorporate clinical examination for assessment of haemorrhoids and CT scan, with most patients being managed conservatively followed by outpatient colonoscopy. Inpatient FS for LGIB in the majority is not recommended. Further studies and clear national guidance is required. 